\title{
Circulating hematopoietic stem cells in patients with choroidal neovascularization secondary to pathologic myopia
}

Department of

Ophthalmology and Visual Sciences, Kyoto University Graduate School of

Medicine, Kyoto, Japan

Correspondence: A Otani, Department of

Ophthalmology and Visual Sciences,

Kyoto University Graduate School of Medicine,

54 Shogoin-Kawahara-cho, Sakyo-ku, Kyoto 606-8386, Japan.

Tel: + 81757513 253;

Fax: + 81757520 933;

E-mail: otan@

kuhp.kyoto-u.ac.jp

Received: 10 January 2008 Accepted in revised form: 29 May 2008

Published online: 20 June 2008

Conflict of interest: None

Financial support: This study was supported by a grantin-aid from the Ministry of Education, Science, Sports and Culture, Japan

\section{Abstract}

Background Emerging evidences suggest that circulating hematopoietic stem cells (HSCs) affect the pathogenesis of choroidal neovascularization (CNV), however, the roles of HSCs in CNV remain unclear in human population. The current study was designed to investigate the role of HSCs in the pathogenesis of CNV secondary to pathologic myopia (PM).

Methods We clinically documented 78 patients with CNV in PM, and 35 of 78 patients and 28 age-matched controls were experimentally analysed. Functional analyses of HSCs were performed using an ex vivo culture system.

Results We disclosed colony-forming units of endothelial cell (CFU-EC) were markedly lower in patients with bilateral CNV compared to those with unilateral CNV (13.8 \pm 3.7 vs $45.9 \pm 7.8, P<0.001)$. Systemic characteristics between both groups showed no significant difference. To identify local ocular factors that may affect the occurrence of $\mathrm{CNV}$, clinical parameters were compared with the following groups in all enrolled subjects: eyes with CNV vs without CNV in unilateral affected patients, and primary affected eyes $v s$ secondary affected eyes in patients with bilateral CNV. However, no statistically significant factors were identified in any of the groups.

Conclusions Circulating HSCs may play a role in the bilateral involvement of $\mathrm{CNV}$ in PM patients as one of the systemic factors. Further prospective and longitudinal studies are required.

Eye (2009) 23, 718-726; doi:10.1038/eye.2008.192; published online 20 June 2008
M Sasahara, A Otani, Y Yodoi, N Gotoh, T Kameda and $\mathrm{N}$ Yoshimura
Keywords: hematopoietic stem cells; choroidal neovascularization; pathologic myopia

Choroidal neovascularization (CNV) is a common pathological change of various chorioretinal diseases ${ }^{1-3}$ that causes severe vision loss. Recently, several new therapeutic options including photodynamic therapy ${ }^{4}$ and anti-vascular endothelial growth factor (VEGF) drugs $^{5}$ have been developed and many investigators have shown the benefits. However, the pathogenesis of CNV is not fully understood and clinically, CNV is still a formidable adversary.

Because there was little information available about the systemic factors that may affect the pathogenesis of $\mathrm{CNV}$, current therapeutic approaches have been designed to control the local factors. Recently, circulating hematopoietic stem cells (HSCs) and endothelial progenitor cells (EPCs) have been reported to incorporate into retinal ${ }^{6}$ and choroidal angiogenic vasculature in murine models. ${ }^{7,8}$ HSCs/EPCs derived from bone marrow ${ }^{9}$ participate in normal and pathological postnatal angiogenesis ${ }^{10}$ and differentiate into a variety of non-endothelial cell (EC) types, including hepatocytes ${ }_{1}^{11}$ cardiomyocytes ${ }^{12}$ microglia, ${ }^{13}$ and even neurons. ${ }^{14}$ It has been suggested that these cells play important roles in repairing injured tissue ${ }^{15,16}$ and disease prognosis. ${ }^{17}$ Although HSCs /EPCs might work as a systemic factor, the role of these cells in the formation of $\mathrm{CNV}$ remains unclear.

By analysing the circulating HSCs with clinical characteristics of age-related macular degeneration (AMD) patients, we have proposed an important role of these cells in 
AMD; the active CNV could increase the number of HSCs in peripheral circulation and the function of these cells might play a key role in the CNV severity and progression. $^{18}$

In this article, we have firstly tried to clarify the roles of HSCs in CNV secondary to pathologic myopia (PM). PM is one of the major leading causes of legal blindness in many countries, especially in Asia including Japan ${ }^{19-22}$ and $\mathrm{CNV}$ is the most important factor on visual prognosis in PM. ${ }^{23,24}$ However, little is known about the pathogenesis. In contrast to the AMD, PM have variety of distinct and characteristic chorioretinal changes including posterior staphyloma, lacquer crack, diffuse chorioretinal atrophy, or patchy chorioretinal atrophy. ${ }^{20}$ Because these local changes have been considered to play important roles in the development of $\mathrm{CNV}$, we considered that analysing those fundus changes with the data of circulating HSCs in PM patients could bring us some important information about the involvement of local and systemic factors in the pathogenesis of CNV. To the best of our knowledge, this is the first report of comprehensive investigation into the local and systemic factors that may affect CNV formation in PM.

\section{Materials and methods}

The study protocol was approved by Ethical Committee of Kyoto University Hospital and all the enrolled subjects gave their written informed consent according to the Declaration of Helsinki. From November 2004 to May 2006, consecutive 101 patients with CNV secondary to PM were diagnosed by comprehensive fundus examinations, including slit-lamp biomicroscopy with a contact lens, fluorescein angiography (FA),

indocyaninegreen angiography (ICGA), and optical coherence tomography (OCT) at Center for Macular Diseases in Kyoto University Hospital. FA and ICGA were simultaneously performed using confocal scanning system (HRA-2, Heidelberg Engineering, Dossenheim, Germany) and two types of OCT were used (Stratus OCT: Carl Zeiss, Dublin, CA, USA; OCT-ophthalmoscope; Nidek, Gamagori, Japan). Inclusion criteria of this study were as follows; (1) Both eyes were high myopia defined as more than $-6.5 \mathrm{D}$ or axial length of $26.5 \mathrm{~mm}$. (2) Intensive clinical examinations including FA, ICGA and OCT were performed in both eyes. (3) Patients having CNV at least in one eye. Seventy-eight of 101 patients satisfied the criteria. The current study is cross-sectional. $\mathrm{CNV}$ location and size was evaluated using fluorescein angiogram and ICG angiogram. In particular, CNV size was defined as the maximal diameter of fibrovascular tissue, and did not include chorioretinal atrophy around CNV membrane. Fundus changes of PM such as diffuse chorioretinal atrophy, patchy chorioretinal atrophy, or chorioretinal atrophy of the macula were evaluated by fundus photograph according to Tokoro's classification. ${ }^{20}$ Diffuse chorioretinal atrophy appears in the posterior pole as a yellowish-white fundus change (Supplementary Figure 1A). In this paper, diffuse chorioretinal atrophy includes spotty or linear lesions with the choroidal vessels. Lesions across choroidal vessels are excluded, because we consider that lesions across choroidal vessels are similar pathologic changes to lacquer crack. Patchy chorioretinal atrophy was subdivided into two groups $\left(P_{1}\right.$ and $\left.P_{2}\right) . P_{1}$ was defined as spotty lesion of the patchy atrophy, $0.6-0.8 \mathrm{~mm}$, less than 0.5 optic disc diameter. $P_{2}$ was defined as patchy lesion of the patchy atrophy, a well-defined round or oval lesion larger than 0.5 optic disc diameter (Supplementary Figure 1B). Posterior staphyloma was observed by indirect ophthalmoscopy (Supplementary Figure 1C). Lacquer crack was observed as hypofluorescent linear lesion imaged by the late phase of ICGA, because ICGA reveals the size, number, and extent of lacquer cracks more clearly than slit-lamp biomicroscopy or FA (Supplementary Figure 1D). ${ }^{25}$

For ex vivo analysis, we randomly collected $20 \mathrm{ml}$ of peripheral blood from 35 of 78 patients who were included in the criteria described above and from 28 agematched controls. The person who had high myopia, macular diseases, other ocular diseases except senile cataract, or systemic diseases such as, diabetes mellitus, cardiovascular diseases, or cerebrovascular diseases was not included in the controls. We mainly investigated colony-forming units of EC (CFU-EC, Hill's assay, Figure 1) and migration activity of HSC-derived cells as the representatives of functional activities of HSCs,

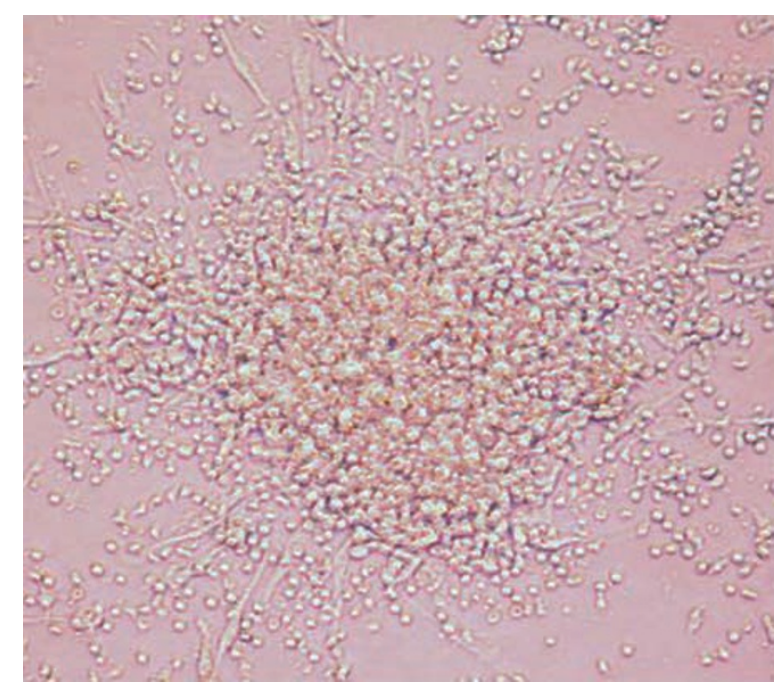

Figure 1 Endothelial colony derived from circulating hematopoietic stem cells. 
because most important pathological event of $\mathrm{CNV}$ formation is angiogenesis. We also analysed the number of circulating HSCs (CD34 + cells) using flow cytometry.

\section{Colony-forming units assay}

Culture of HSCs and measuring CFUs were performed using an established method previously described by Hill et al. ${ }^{26}$ Mononuclear cells from peripheral blood were collected by light-density gradient centrifugation (FicollPaque Plus, Amersham Biosciences) and $1 \times 10^{7}$ of mononuclear cells were seeded on 6-well human fibronectin-coated plates (BD Biosciences) in a $2.5 \mathrm{ml}$ of an endothelial basal medium (EBM) (Endocult, StemCell Technologies) with $20 \%$ fetal bovine serum (FBS). After $48 \mathrm{~h}, 1 \times 10^{6}$ non-adherent cells were transferred into a new 24-well fibronectin coated plates in $1 \mathrm{ml}$ of EBM to avoid contamination with mature ECs and nonprogenitor cells and incubated for another 3 days. After 5 days culture in vitro, endothelial colonies of more than 50 cells in two wells were counted by two independent investigators. Mean value of three wells was scored as CFUs. Immunocytochemistry was performed after fixation of $4 \%$ paraformaldehyde, and the expression of endothelial marker proteins, kinase insert domain receptor (R\&D Systems), platelet EC adhesion molecule-1 (R\&D Systems), vascular endothelial-cadherin (Chemicon) was confirmed.

\section{Migration assay of hematopoietic stem cell-derived cells}

HSC-derived cells after 5 days culture in vitro were placed in the upper chamber $\left(2.5 \times 10^{4}\right)$ of a modified Boyden chamber with a fluorescence block system ${ }^{27}$ (BD Biocoat ${ }^{\mathrm{TM}}$ Angiogenesis System: Endothelial Cell Migration, BD Biosciences) in duplicate for each patient's sample. The chamber was placed in a 96-well culture dish containing EBM (Endocult, StemCell Technologies) and $10 \%$ FBS. After $22 \mathrm{~h}$ incubation for $37^{\circ} \mathrm{C}$, the lower side of the filter was washed and the migrated cells were stained with a fluorogenic esterase substrate (Calcein AM, Molecular Probes). Migration activity was measured using a fluorescent plate reader with a bottom-reading system (ARVO, PerkinElmer). Data were presented as percentage of relative fluorescence units with chemoattractants to without chemoattractant (EBM only).

\section{The number of circulating hematopoietic stem cells}

The number of circulating CD34 + HSCs was counted using flow cytometric analysis. ${ }^{18}$ Circulating CD34+ mononuclear cells in the peripheral blood were stained using a reagent kit (ProCOUNT, BD Biosciences, Bedford, MA, USA) and measured with a flow cytometer
(FACSCalibur, BD). The known amount of stained and lysed, but not washed, blood added to a known amount of microbeads allows, in combination with the observed ratio between the number of flow cytometrically counted beads and CD34 + cells, the calculation of absolute CD34 + cell count. The data were analysed using Cell Quest software (BD Biosciences). In each blood sample, more than 60000 cell events were obtained. An isotypenegative control optimized the settings of the fluorescence detectors for each subject.

\section{Statistical analysis}

All results for continuous variables in the experimental data are expressed as means \pm SEM. Comparisons between groups were analysed by paired or unpaired Student's $t$-test (two-sided) for parametric analysis. For non-parametric analysis, Mann-Whitney's $U$-test was used. Categorical analysis was calculated by $\chi^{2}$ test and Fisher's exact test. Multiple regression analysis was performed to correlate CFU-EC with the systemic risk factors or clinical conditions. $P<0.05$ was considered statistically significant. All analyses were performed with SPSS 13.0 software.

\section{Results}

\section{Patient backgrounds}

Seventy eight patients were included in this study. The average age was $64.8 \pm 11.5$ years (range 22-84 years). Eight patients (10\%) were less than 50 years old; 14 (18\%) were male and $64(82 \%)$ were female.

Majority of the subjects had active CNV lesion, which was demonstrated by significant fluorescein leakage on angiogram, haemorrhage, and cystoid macular oedema observed by OCT. Patients who underwent photodynamic therapy or intravenous steroid injection within 6 months and within 3 months were $89 \%(n=31)$ and $74 \%(n=26)$, respectively.

\section{Clinical evaluation}

To find a local ocular factor, which may affect the development of $\mathrm{CNV}$, we compared the clinically documented fundus changes in each eye of an individual patient. The first investigation was the comparison of fundus appearance between the affected eye and nonaffected eye in unilateral CNV patients. In this investigation, we have focused on a question whether the affected eye had severer pathologic changes than the non-affected eye. The second investigation was in the bilateral affected patients and the severity of PM changes was compared between a primary affected eye and a 
secondary affected eye. To achieve these investigations, the all enrolled patients $(n=78)$ were divided into unilateral (57 patients, $73 \%$ ) and bilateral (21 patients, $27 \%$ ) CNV affected groups. Table 1 shows the comparison of clinical parameters between an affected eye and non-affected eye in patients of unilateral involvement. As expected, visual acuity (VA) of the eyes with CNV was significantly worse than the eyes without $\mathrm{CNV}$. There was no significant difference in many of the clinical parameters examined, which may reflect the severity of PM, such as axial length, posterior

Table 1 Comparison of clinical characteristics between eyes with and without choroidal neovascularization with unilateral involvement in pathologic myopia

\begin{tabular}{|c|c|c|c|}
\hline & $\begin{array}{c}\text { Eyes with } \\
\text { CNV } \\
(\mathrm{n}=57)\end{array}$ & $\begin{array}{c}\text { Eyes without } \\
\text { CNV } \\
(\mathrm{n}=57)\end{array}$ & P-value \\
\hline \multicolumn{4}{|l|}{ Refraction (D) } \\
\hline Mean \pm SD & $-11.48 \pm 4.63$ & $-11.35 \pm 4.09$ & 0.074 \\
\hline Range & -4.0 to -22.0 & -4.25 to -20.0 & \\
\hline \multicolumn{4}{|l|}{ Keratometry (mm) } \\
\hline Mean \pm SD & $7.60 \pm 0.27$ & $7.62 \pm 0.27$ & 0.83 \\
\hline Range & $6.82-8.09$ & $6.85-8.25$ & \\
\hline \multicolumn{4}{|l|}{ Axial length (mm) } \\
\hline Mean \pm SD & $28.86 \pm 1.31$ & $28.60 \pm 1.63$ & 0.35 \\
\hline Range & $26.35-32.50$ & $24.43-31.25$ & \\
\hline \multicolumn{4}{|l|}{ Visual acuity } \\
\hline $20 / 200-20 / 40$ & $31(54 \%)$ & $11(19 \%)$ & $<0.001$ \\
\hline$<20 / 200$ & $14(25 \%)$ & $5(8.8 \%)$ & 0.04 \\
\hline \multicolumn{4}{|l|}{ CNV location } \\
\hline Subfoveal & $31(54 \%)$ & - & \\
\hline Juxtafoveal & $24(42 \%)$ & - & \\
\hline Extrafoveal & $2(3.5 \%)$ & - & \\
\hline \multicolumn{4}{|l|}{ CNV size $(\mu m)$} \\
\hline Mean \pm SD & $1492 \pm 811$ & - & \\
\hline Range & $350-4130$ & - & \\
\hline Posterior staphyloma & $24 / 26(92 \%)$ & $20 / 26(77 \%)$ & 0.25 \\
\hline Diffuse atrophy & $51(89 \%)$ & $42(74 \%)$ & 0.051 \\
\hline \multicolumn{4}{|l|}{ Patchy atrophy } \\
\hline$P_{1}$ & $9(16 \%)$ & $11(19 \%)$ & 0.81 \\
\hline$P_{2}$ & $6(11 \%)$ & $8(14 \%)$ & 0.78 \\
\hline MA & $6(11 \%)$ & $4(7.0 \%)$ & 0.74 \\
\hline \multicolumn{4}{|l|}{ Lacquer crack } \\
\hline Detected in ICGA ${ }^{a}$ & $50 / 55(91 \%)$ & $44 / 54(81 \%)$ & 0.18 \\
\hline Not applicable & $2(3.5 \%)$ & $3(5.3 \%)$ & \\
\hline
\end{tabular}

$\mathrm{CNV}=$ choroidal neovascularization; ICGA = indocyaninegreen angiography; $\mathrm{MA}=$ chorioretinal atrophy of the macula; $\mathrm{P}_{1=}$ spotty lesion of the patchy atrophy; $\mathrm{P}_{2}=$ patchy lesion of the patchy atrophy.

${ }^{a}$ Not applicable eyes were excluded. staphyloma, patchy chorioretinal atrophy, or lacquer cracks. But, diffuse chorioretinal atrophy was more frequently seen in the eyes with CNV (89\%) compared to the contra lateral eyes without $\mathrm{CNV}(74 \%)$ with borderline significance $(P=0.051)$. Table 2 shows the comparison of clinical parameters between a primary affected eye and a secondary affected eye in patients of bilateral involvement. The size of CNV was significantly larger in primary affected eyes than the secondary affected eyes $(P=0.02)$. Similar to the analysis of unilateral $\mathrm{CNV}$ affected cases, many of the fundus

Table 2 Clinical characteristics of patients with bilateral myopic choroidal neovascularization: comparison of primary and secondary affected eyes

\begin{tabular}{|c|c|c|c|}
\hline & $\begin{array}{c}\text { Primary } \\
\text { affected } \\
\text { eyes }(\mathrm{n}=21)\end{array}$ & $\begin{array}{c}\text { Secondary } \\
\text { affected } \\
\text { eyes }(\mathrm{n}=21)\end{array}$ & P-value \\
\hline \multicolumn{4}{|l|}{ Refraction (D) } \\
\hline Mean \pm SD & $-10.88 \pm 3.32$ & $-10.40 \pm 2.95$ & 0.14 \\
\hline Range & -4.25 to -17.0 & -5.25 to -10.50 & \\
\hline \multicolumn{4}{|l|}{ Keratometry (mm) } \\
\hline Mean \pm SD & $7.50 \pm 0.33$ & $7.38 \pm 0.38$ & 0.64 \\
\hline Range & $7.05-8.11$ & $6.59-7.96$ & \\
\hline \multicolumn{4}{|l|}{ Axial length (mm) } \\
\hline Mean \pm SD & $28.53 \pm 1.42$ & $28.59 \pm 1.65$ & 0.91 \\
\hline Range & $26.38-32.63$ & $24.66-32.58$ & \\
\hline \multicolumn{4}{|l|}{ Visual acuity } \\
\hline $20 / 200-20 / 40$ & $9(43 \%)$ & $14(67 \%)$ & 0.35 \\
\hline$<20 / 200$ & $9(43 \%)$ & $2(9.5 \%)$ & 0.053 \\
\hline \multicolumn{4}{|l|}{ CNV location } \\
\hline Subfoveal & $18(86 \%)$ & $13(62 \%)$ & 0.16 \\
\hline Juxtafoveal & $3(14 \%)$ & $7(33 \%)$ & 0.28 \\
\hline Extrafoveal & $0(0 \%)$ & $1(4.8 \%)$ & - \\
\hline \multicolumn{4}{|l|}{ CNV size $(\mu \mathrm{m})$} \\
\hline Mean \pm SD & $2444 \pm 1029$ & $1772 \pm 817$ & 0.02 \\
\hline Range & $1160-5220$ & $610-3930$ & \\
\hline Posterior staphyloma & $11 / 11(100 \%)$ & $11 / 11(100 \%)$ & 1.0 \\
\hline Diffuse atrophy & $21(100 \%)$ & $21(100 \%)$ & 1.0 \\
\hline \multicolumn{4}{|l|}{ Patchy atrophy } \\
\hline$P_{1}$ & $4(19 \%)$ & $7(33 \%)$ & 0.48 \\
\hline$P_{2}$ & $5(24 \%)$ & $5(24 \%)$ & 1.0 \\
\hline MA & $15(63 \%)$ & $0(0 \%)$ & $<0.001$ \\
\hline \multicolumn{4}{|l|}{ Lacquer crack } \\
\hline Detected in ICGA ${ }^{a}$ & $11 / 12(92 \%)$ & $20 / 21(95 \%)$ & 0.38 \\
\hline Not applicable & $9(43 \%)$ & $0(0 \%)$ & \\
\hline
\end{tabular}

$\mathrm{CNV}=$ choroidal neovascularization; ICGA = indocyaninegreen angiography; $\mathrm{MA}=$ chorioretinal atrophy of the macula; $P_{1}=$ spotty lesion of the patchy atrophy; $P_{2}=$ patchy lesion of the patchy atrophy.

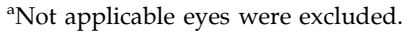


changes did not show significant difference between the primary and secondary $\mathrm{CNV}$ eyes; however, the chorioretinal atrophy of the macula $(P<0.001)$ is significantly frequently seen in primary affected eyes.

\section{Functional evaluation of HSCs}

Thirty-five PM patients with CNV were enrolled to the ex vivo analysis of circulating HSCs. Table 3 shows systemic characteristics of these patients and age-matched non-PM controls. Details of analysed data are shown in Table 4. The average CFU-EC or migration activity of myopic $\mathrm{CNV}$ patients $(n=35)$ did not significantly different to that of age-matched controls ( $n=28$, Figure $2 a$ and $b)$. The number of circulating CD34 + HSCs also

Table 3 Systemic characteristics of subjects used for HSC analysis: myopic CNV vs control

\begin{tabular}{lccc}
\hline & $\begin{array}{c}\text { Myopic CNV } \\
(\mathrm{n}=35)\end{array}$ & $\begin{array}{c}\text { Controls } \\
(\mathrm{n}=28)\end{array}$ & P-value \\
\hline Age (years) & & & \\
$\quad$ Mean \pm SD & $66.3 \pm 13.3$ & $65.6 \pm 3.6$ & 0.86 \\
$\quad$ Range & $27-82$ & $26-87$ & \\
Gender (female) & $30(86 \%)$ & $21(75 \%)$ & 0.45 \\
Hypertension & $9(26 \%)$ & $6(21 \%)$ & 0.92 \\
Diabetes mellitus & $2(5.7 \%)$ & $4(14 \%)$ & 0.39 \\
Hyperlipidemia & $11(31 \%)$ & $7(25 \%)$ & 0.78 \\
Statin medication & $6(17 \%)$ & $3(11 \%)$ & 0.72 \\
Cardiovasular disease & $7(20 \%)$ & $4(14 \%)$ & 0.74 \\
Cerebrovascular disease & $0(0 \%)$ & $1(3.6 \%)$ & 0.44 \\
History of smoking & $8(23 \%)$ & $5(18 \%)$ & 0.86 \\
\hline
\end{tabular}

$\mathrm{CNV}=$ choroidal neovascularization, $\mathrm{HSC}=$ hematopoietic stem cell.

Table 4 Data of HSC analysis

\begin{tabular}{|c|c|c|c|c|}
\hline & \multirow{2}{*}{$\begin{array}{l}\text { Controls } \\
(\mathrm{n}=28)\end{array}$} & \multicolumn{3}{|c|}{ Myopic CNV } \\
\hline & & $\begin{array}{l}\text { Overall } \\
(\mathrm{n}=35)\end{array}$ & $\begin{array}{l}\text { Unilateral } \\
(\mathrm{n}=27)\end{array}$ & $\begin{array}{c}\text { Bilateral } \\
(\mathrm{n}=8)\end{array}$ \\
\hline \multicolumn{5}{|l|}{ CFU-EC } \\
\hline Mean \pm SEM & $39.4 \pm 5.1$ & $41.3 \pm 5.7$ & $49.5 \pm 7.8$ & $13.8 \pm 3.7$ \\
\hline Range & $0.5-115.0$ & $1.0-138.0$ & $1.0-138.0$ & $1.0-33.5$ \\
\hline Median & 39.0 & 33.5 & 46.5 & 13.3 \\
\hline \multicolumn{5}{|c|}{ Migration (percentage of basal) } \\
\hline Mean \pm SEM & $155.1 \pm 5.4$ & $147.9 \pm 4.4$ & $150.7 \pm 8.3$ & $138.3 \pm 7.8$ \\
\hline Range & $112-216$ & 105-207 & $112-207$ & 105-175 \\
\hline Median & 155.0 & 144.0 & 146.0 & 136.0 \\
\hline \multicolumn{5}{|c|}{ 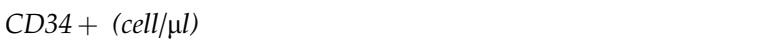 } \\
\hline Mean \pm SEM & $3.95 \pm 0.43$ & $4.55 \pm 0.37$ & $4.56 \pm 0.47$ & $4.53 \pm 0.61$ \\
\hline Range & $1.49-8.67$ & $1.67-8.61$ & $1.66-8.61$ & $1.92-6.70$ \\
\hline Median & 3.95 & 4.53 & 4.59 & 4.41 \\
\hline
\end{tabular}

showed no difference between the patients and controls (Figure 2c). We then analysed whether there are any association between clinical characteristics and functional activities of circulating HSCs. Examined clinical parameters were refraction, axial length, corrected VA, CNV location, CNV size, laterality of CNV, diffuse chorioretinal atrophy, patchy atrophy, lacquer cracks, and posterior staphyloma. Among the various combinations examined, we found that CFU-EC of bilateral CNV patients showed markedly lower than those of unilateral CNV patients ( $P<0.001$, Figure $2 \mathrm{~d}$ ). In addition, the migration activity of HSCs was also reduced by $24 \%$ in bilateral patients compared to unilateral patients (Figure 2e). No difference was observed in systemic characteristics between the bilateral and unilateral groups (Table 5). Multiple regression analysis also showed that this result in function may not be brought by these systemic factors (Table 6). In contrast, the number of circulating CD34 + HSCs also showed no difference between the unilateral and bilateral patients (Figure 2f).

\section{Discussion}

$\mathrm{CNV}$ is the most common cause of severe vision loss in PM same as in AMD. Although there are several differences in the clinical characteristics between AMD and PM the pathogenesis of $\mathrm{CNV}$ are considered same; neovascularization originate from choroidal vasculature that grown through the break of Bruch's membrane. Studies on the histopathology of CNV also suggested that there are no significant differences in the constituent cells and in the expression of several important cytokines including VEGF-A. ${ }^{28,29}$ One of the characteristic features of PM patients that differ from AMD patients is the progressive elongation of the eyeball that results in posterior staphyloma. Within the posterior staphyloma, many characteristic fundus changes including diffuse atrophy of retina and choroid, patchy chorioretinal atrophy, and lacquer cracks are observed. Because these pathologic changes possibly result in the breaks of Bruch's membrane, it is reasonable that the development of $\mathrm{CNV}$ have correlation with these changes. ${ }^{20,30,31}$ Another characteristic of PM patients is the younger age. In contrast to AMD patients, $\mathrm{CNV}$ can be developed in the younger patients in PM and it is reported that PM is the most major cause of $\mathrm{CNV}$ development in the young generation. ${ }^{2}$ This fact may also be explained by the chorioretinal changes of PM. Indeed, as the possible developmental factors of myopic CNV, increasing age, ${ }^{20}$ axial length, ${ }^{20}$ lacquer crack, ${ }^{30,31}$ and patchy atrophy ${ }^{31}$ have been reported.

To investigate whether and how these local ocular factors are involved in the development of myopic CNV, 

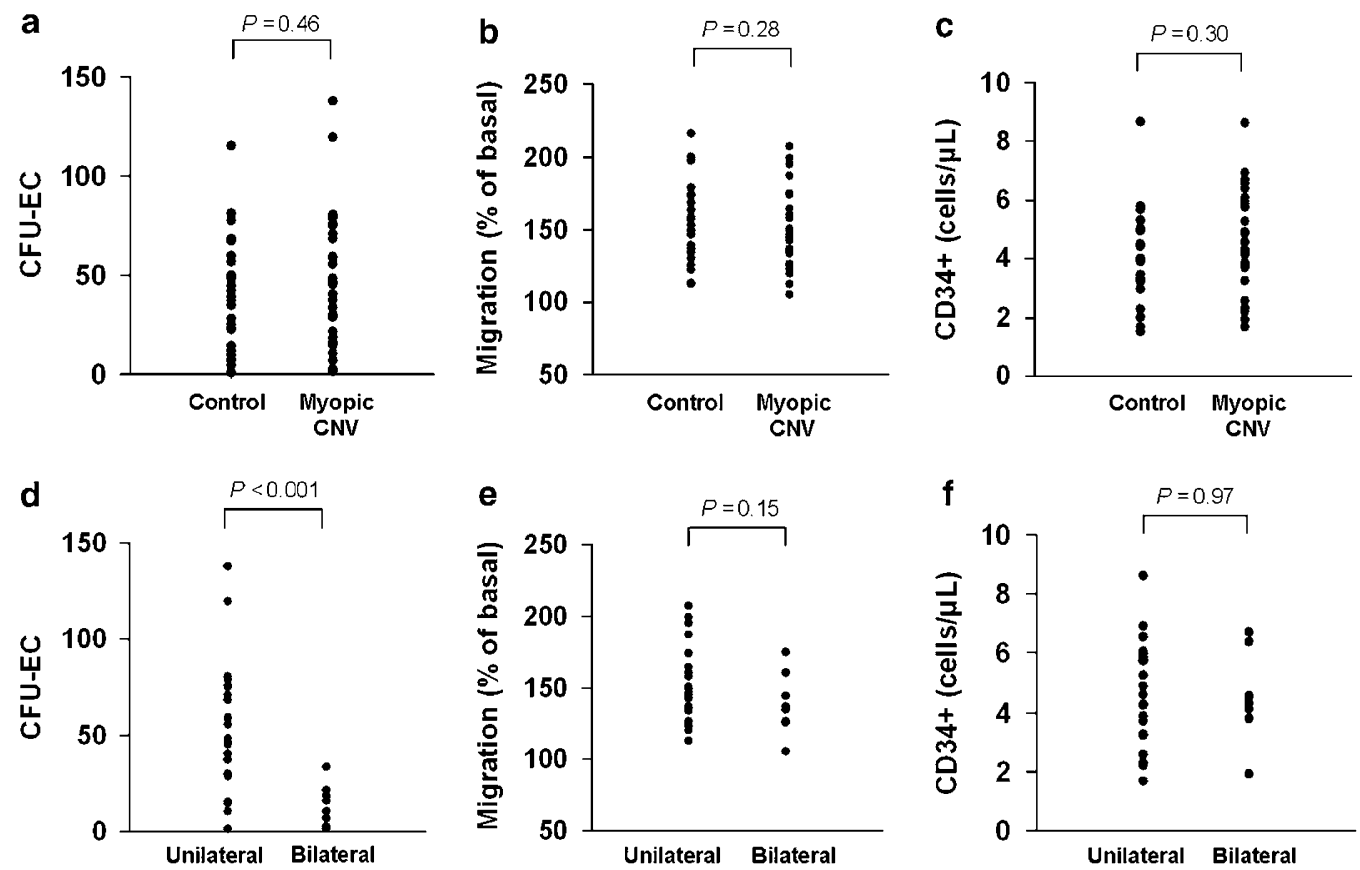

Figure 2 Analyses of the functions and numbers of circulating hematopoietic stem cells (HSCs) in patients with choroidal neovascularization (CNV) secondary to pathologic myopia (PM). (a) Comparison of colony-forming units of endothelial cell (CFU-EC) between the age-matched healthy controls $(n=28)$ and patients with CNV secondary to PM $(n=35)$. (b) Comparison of migration activity between the controls $(n=28)$ and patients $(n=35)$. (c) Comparison of the number of circulating CD34 + HSCs between the age-matched healthy controls $(n=28)$ and the patient with CNV secondary to PM $(n=35)$. (d) Impaired CFU-EC in patients with bilateral CNV $(n=8)$ compared to those with unilateral CNV $(n=27)$. (e) Reduced migration activity of endothelial progenitor cells in patients with bilateral CNV $(n=8)$ compared to those with unilateral CNV ( $n=27)$. (f) No significant difference in the number of circulating CD34 + HSCs between patients with unilateral $(n=27)$ and bilateral $(n=8) \mathrm{CNV}$.

Table 5 Systemic characteristics of subjects used for HSC analysis: unilateral vs bilateral

\begin{tabular}{lcll}
\hline & $\begin{array}{c}\text { Unilateral } \\
(\mathrm{n}=27)\end{array}$ & $\begin{array}{c}\text { Bilateral } \\
(\mathrm{n}=8)\end{array}$ & P-value \\
\hline Age (years) & & & \\
$\quad$ Mean \pm SD & $65.3 \pm 14.3$ & $69.9 \pm 8.7$ & 0.40 \\
$\quad$ Range & $27-82$ & $56-78$ & \\
Gender (female) & $22(81 \%)$ & $8(100 \%)$ & 0.28 \\
Hypertension & $9(33 \%)$ & $0(0 \%)$ & 0.081 \\
Diabetes mellitus & $2(5.7 \%)$ & $0(0 \%)$ & 1.0 \\
Hyperlipidemia & $9(26 \%)$ & $2(25 \%)$ & 1.0 \\
Statin medication & $3(11 \%)$ & $1(13 \%)$ & 1.0 \\
Cardiovasular disease & $6(17 \%)$ & $1(13 \%)$ & 1.0 \\
Cerebrovascular disease & $0(0 \%)$ & $0(0 \%)$ & 1.0 \\
History of smoking & $6(17 \%)$ & $2(25 \%)$ & 1.0 \\
\hline
\end{tabular}

$\mathrm{HSC}=$ hematopoietic stem cell.

we first examined the funduscopic differences between the eyes of an individual patient. By comparing each eye of individual patient, it was possible to ignore the influences of systemic factors and we thought that the local ocular factors that affect the pathogenesis of $\mathrm{CNV}$ might be brought into relief. However, unexpectedly, there were no funduscopic differences between the affected eyes and the non-affected eyes in unilateral CNV patients. In addition, we could find no significant difference except diffuse chorioretinal atrophy of the macula in those between the primary affected eyes and the secondary affected eyes of bilateral CNV affected patients. These data do not deny the importance of pathologic changes of PM in the development of CNV; however, our results may indicate that these characteristic pathological changes in PM is a necessary condition, but not a sufficient condition, in CNV development. Other conditions may be required to develop CNV in the eyes of PM.

Circulating HSCs have been shown to involve in the pathogenesis of $\mathrm{CNV}$ in murine laser-induced $\mathrm{CNV}$ model. ${ }^{78}$ From the previous functional analysis of circulating HSCs in the neovascular AMD patients, we disclosed functional impairments of HSCs in patients with severer $\mathrm{CNV}$, and revealed that HSCs may have a protective role against $\mathrm{CNV}$ progression. ${ }^{18}$ Similar to the data of AMD patients, our data on functional analysis of HSCs suggest that circulating bone marrow-derived stem cells may contribute to the pathogenesis of myopic CNV as one of the systemic factors. Among several combinations, we found that the laterality of $\mathrm{CNV}$ is 
Table 6 Multiple regression analysis between CFU-EC and clinical conditions

\begin{tabular}{lcc}
\hline Independent variable & $\begin{array}{c}\beta \text { coefficient } \\
(\mathrm{R}=0.91)\end{array}$ & P-value \\
\hline HSC analysis & & \\
Migration & 0.51 & 0.04 \\
CD34 + cells & 0.28 & 0.27 \\
Clinical charateristics & & \\
Age & & \\
Sex (male) & 0.05 & 0.85 \\
Refraction & -0.29 & 0.31 \\
Keratometry & -0.17 & 0.47 \\
Axial length & 0.00 & 1.00 \\
Corrected visual acuity & -0.26 & 0.30 \\
Laterality of CNV (bilateral) & 0.07 & 0.70 \\
CNV location (subfoveal) & -0.14 & 0.76 \\
CNV size & 0.38 & 0.08 \\
Posterior staphyloma & -0.38 & 0.32 \\
Diffuse atrophy & -0.01 & 0.97 \\
Patchy atrophy & -0.21 & 0.29 \\
& -0.14 & 0.50 \\
Systemic characteristics & & \\
Hypertension & & \\
Diabetes mellitus & 0.27 & 0.38 \\
Hyperlipidemia & -0.11 & 0.54 \\
Heart disease & -0.12 & 0.57 \\
Medication of anticoagulants & 0.45 & 0.09 \\
History of smoking & 0.30 & 0.19 \\
\hline CNV cho & -0.33 & 0.23 \\
\hline
\end{tabular}

$\mathrm{CNV}=$ choroidal neovascularization; $\mathrm{CFU}-\mathrm{EC}=$ colony-forming units of endothelial cell; HSC = hematopoietic stem cell.

related to the functional capacities of HSCs. In bilateral affected patients, functional activities of HSCs were significantly reduced compared to the unilateral affected patients. These data are the first clinical evidence to demonstrate the roles of circulating HSCs for CNV in PM as one of the systemic factors.

Recent study on CNV model reports that circulating HSCs regenerate the injured retinal pigment epithelial (RPE) cells or other type of cells (astrocyte, macrophage/ microglia, mural cells, and vascular ECs). ${ }^{32}$ The study also suggests that circulating HSCs have a repairing capacity for chronic injured RPE or neurosensory retina. Similar paradigm was already proposed in the studies dealing with atherosclerosis. ${ }^{15,33}$ EPCs derived from HSCs are considered to have the capacity to repair vascular injuries. The injured endothelial monolayer is regenerated by circulating EPCs, accelerating reendothelialization and limiting atherosclerotic lesion formation. Moreover, EPC impairment by risk factors, such as age and diabetes, may contribute to atherogenesis and atherosclerotic disease progression. Thus, a possible role of circulating HSCs may be a tissue repair for tissue injuries in highly myopic eyes, and we speculate that impaired functional HSCs cannot maintain subclinical tissue injuries, resulting in having high risks for CNV development and bilateral involvement. To strengthen this hypothesis, it is necessary to correct the blood samples from myopic without CNV persons. Our result was that CFU-EC showed no significant difference between the non-myopic control subjects and the PM patients with CNV (Figure $2 a$ and $b$ ). If CFU-EC or the migration activity of myopic without $\mathrm{CNV}$ people is higher than unilateral $\mathrm{CNV}$ patients, the functional capacities of HSCs might be a risk factor for CNV in PM. Another limitation of this study is the small number of bilateral patients who were experimentally analysed $(n=8)$. A larger population study is required. In addition, CNV size was not related to HSC functions in PM patients. Contrastively, AMD patients with larger CNV had reduced functions of HSCs. ${ }^{18}$ This difference may be originated from the small number in this study or may show the different role of circulating HSCs between the development and the progression of CNV in two different ocular environments (AMD and $\mathrm{PM})$.

We have recently reported that the number of HSCs was significantly increased with the activity of CNV in neovascular AMD patients and showed a possibility that active CNV lesion can mobilize HSCs from bone marrow. ${ }^{18}$ To investigate the role of HSCs, the number of cells involved is another important factor that should be considered in addition to the function of cells. Although the number of HSCs in myopic CNV patients was higher than that of healthy controls, statistically significant difference was not present in our study population (Figure 2c). Furthermore, probably because the majority of both unilateral and bilateral patients had active CNV lesion, the number of HSCs showed no difference between the two groups in contrast to the function of HSCs. Thus, the functional difference rather than the number of BM-derived HSCs may be a clue in making the clinical differences in our myopic $\mathrm{CNV}$ cases; however, requirement of further study is beyond doubt.

In conclusion, we have investigated the funduscopic chorioretinal changes as local factors and circulating BMderived stem cells as systemic factors whether they have any role in the pathogenesis of CNV in PM patients. Although we could not find local ocular factors that can affect the laterality of myopic $\mathrm{CNV}$, impaired function of circulating BM-derived stem cells were observed in patients of bilateral CNV involvement. Because CNV is known to grow through a break of Bruch's membrane, ocular changes including chorioretinal atrophy in PM patients may play an important role in the pathogenesis $\mathrm{CNV}$. However, the reason why not all the PM patients develop CNV in both eyes in spite of the presence of chorioretinal changes is an unsolved question. Our 
results of functional differences of circulating BMderived stem cells in PM patients may suggest a key to solve this question. Further longitudinal clinical studies and experimental studies are expected to clarify the underling mechanism of pathogenesis of CNV in PM patients.

\section{Acknowledgements}

We thank our colleagues and laboratory members for helpful advice and technical support. We specially thank Kayo Nishida for her great technical support. This work supported by a grant-in-aid from the Ministry of Education, Science, Sports and Culture, Japan (No. 17689045).

\section{References}

1 Gass JDM. Stereoscopic Atlas of Macular Diseases, 4th edn. Mosby: St Louis, 1997.

2 Cohen SY, Laroche A, Leguen Y, Soubrane G, Coscas GJ. Etiology of choroidal neovascularization in young patients. Ophthalmology 1996; 103: 1241-1244.

3 Kent D, Sheridan C. Choroidal neovascularization: a wound healing perspective. Mol Vis 2003; 9: 747-755.

4 Schmidt-Erfurth U, Miller J, Sickenberg M, Bunse A, Laqua H, Gragoudas E et al. Photodynamic therapy of subfoveal choroidal neovascularization: clinical and angiographic examples. Graefes Arch Clin Exp Ophthalmol 1998; 236: 365-374.

5 Gragoudas ES, Adamis AP, Cunningham Jr ET, Feinsod M, Guyer DR. Pegaptanib for neovascular age-related macular degeneration. N Engl J Med 2004; 351: 2805-2816.

6 Grant MB, May WS, Caballero S, Brown GA, Guthrie SM, Mames RN et al. Adult hematopoietic stem cells provide functional hemangioblast activity during retinal neovascularization. Nat Med 2002; 8: 607-612.

7 Espinosa-Heidmann DG, Caicedo A, Hernandez EP, Csaky KG, Cousins SW. Bone marrow-derived progenitor cells contribute to experimental choroidal neovascularization. Invest Ophthalmol Vis Sci 2003; 44: 4914-4919.

8 Sengupta N, Caballero S, Mames RN, Butler JM, Scott EW, Grant MB et al. The role of adult bone marrow-derived stem cells in choroidal neovascularization. Invest Ophthalmol Vis Sci 2003; 44:

4908-4913.

9 Asahara T, Murohara T, Sullivan A, Silver M, Van der Zee R, $\mathrm{Li} \mathrm{T}$ et al. Isolation of putative progenitor endothelial cells for angiogenesis. Science 1997; 275: 964-967.

10 Lyden D, Hattori K, Dias S, Costa C, Blaikie P, Butros L et al. Impaired recruitment of bone-marrow-derived endothelial and hematopoietic precursor cells blocks tumor angiogenesis and growth. Nat Med 2001; 7: 1194-1201.

11 Lagasse E, Connors H, Al-Dhalimy M, Reitsma M, Dohse M, Osborne L et al. Purified hematopoietic stem cells can differentiate into hepatocytes in vivo. Nat Med 2000; 6: 12291234.

12 Orlic D, Kajstura J, Chimenti S, Limana F, Jakoniuk I, Quaini F et al. Mobilized bone marrow cells repair the infarcted heart, improving function and survival. Proc Natl Acad Sci USA 2001; 98: 10344-10349.

13 Priller J, Flugel A, Wehner T, Boentert M, Haas CA, Prinz M et al. Targeting gene-modified hematopoietic cells to the central nervous system: use of green fluorescent protein uncovers microglial engraftment. Nat Med 2001; 7: 1356-1361.

14 Shyu WC, Lin SZ, Chiang MF, Su CY, Li H. Intracerebral peripheral blood stem cell $(\mathrm{CD} 34+)$ implantation induces neuroplasticity by enhancing beta1 integrin-mediated angiogenesis in chronic stroke rats. J Neurosci 2006; 26: 3444-3453.

15 Schmidt-Lucke C, Rossig L, Fichtlscherer S, Vasa M, Britten M, Kämper U et al. Reduced number of circulating endothelial progenitor cells predicts future cardiovascular events: proof of concept for the clinical importance of endogenous vascular repair. Circulation 2005; 111: 2981-2987.

16 Kawamoto A, Gwon HC, Iwaguro H, Yamaguchi JI, Uchida $\mathrm{S}$, Masuda $\mathrm{H}$ et al. Therapeutic potential of ex vivo expanded endothelial progenitor cells for myocardial ischemia. Circulation 2001; 103: 634-637.

17 Werner N, Kosiol S, Schiegl T, Ahlers P, Walenta K, Link A et al. Circulating endothelial progenitor cells and cardiovascular outcomes. N Engl J Med 2005; 353: 999-1007.

18 Yodoi Y, Sasahara M, Kameda T, Yoshimura N, Otani A. Circulating hematopoietic stem cells in patients with neovascular age-related macular degeneration. Invest Ophthalmol Vis Sci 2007; 48: 5464-5472.

19 Ghafour IM, Allan D, Foulds WS. Common causes of blindness and visual handicap in the west of Scotland. $\mathrm{Br}$ J Ophthalmol 1983; 67: 209-213.

20 Tokoro T. Atlas of Posterior Fundus Changes in Pathologic Myopia. Springer: Tokyo, 1998.

21 Sperduto RD, Seigel D, Roberts J, Rowland M. Prevalence of myopia in the United States. Arch Ophthalmol 1983; 101: 405-407.

22 Tokoro T. On the definition of pathologic myopia in group studies. Acta Ophthalmol Suppl 1988; 185: 107-108.

23 Avila MP, Weiter JJ, Jalkh AE, Trempe CL, Pruett RC, Schepens CL. Natural history of choroidal neovascularization in degenerative myopia. Ophthalmology 1984; 91: 1573-1581.

24 Hampton GR, Kohen D, Bird AC. Visual prognosis of disciform degeneration in myopia. Ophthalmology 1983; 90: 923-926.

25 Ohno-Matsui K, Morishima N, Ito M, Tokoro T. Indocyanine green angiographic findings of lacquer cracks in pathologic myopia. Jpn J Ophthalmol 1998; 42: 293-299.

26 Hill JM, Zalos G, Halcox JP, Schenke WH, Waclawiw MA, Quyyumi AA et al. Circulating endothelial progenitor cells, vascular function, and cardiovascular risk. $N$ Engl J Med 2003; 348: 593-600.

27 Maliakal JC. Quantitative high throughput endothelial cell migration and invasion assay system. Methods Enzymol 2002; 352: 175-182.

28 Grossniklaus HE, Hutchinson AK, Capone Jr A, Woolfson J, Lambert HM. Clinicopathologic features of surgically excised choroidal neovascular membranes. Ophthalmology 1994; 101: 1099-1111.

29 Otani A, Takagi H, Oh H, Koyama S, Ogura Y, Matumura M et al. Vascular endothelial growth factor family and receptor expression in human choroidal neovascular membranes. Microvasc Res 2002; 64: 162-169.

30 Ohno-Matsui K, Tokoro T. The progression of lacquer cracks in pathologic myopia. Retina 1996; 16: 29-37. 
31 Ohno-Matsui K, Yoshida T, Futagami S, Yasuzumi K, Shimada N, Kojima A et al. Patchy atrophy and lacquer cracks predispose to the development of choroidal neovascularisation in pathological myopia. Br J Ophthalmol 2003; 87: 570-573.

32 Chan-Ling T, Baxter L, Afzal A, Sengupta N, Caballero S, Rosinova E et al. Hematopoietic stem cells provide repair functions after laser-induced Bruch's membrane rupture model of choroidal neovascularization. Am J Pathol 2006; 168: 1031-1044.

33 Dimmeler S, Zeiher AM. Vascular repair by circulating endothelial progenitor cells: the missing link in atherosclerosis? J Mol Med 2004; 82 : 671-677.

Supplementary Information accompanies the paper on Eye website (http://www.nature.com/eye) 